\title{
Immobilization-induced hypercalcemia in a patient with renal failure
}

\author{
Anand Gandhi'1,2, Mike Mortensen 2,3,4, Sonie Sunny²,3,4, Pawarid Techathaveewat 2,3,4, Jerome Targovnik ${ }^{4}$ and \\ Mahmoud Alsayed 2,3
}

${ }^{1}$ Division of Internal Medicine, University of Arizona College of Medicine - Phoenix, Phoenix, Arizona, USA, ${ }^{2}$ Banner University Medical Center - Phoenix, Phoenix, Arizona, USA, ${ }^{3}$ Division of Endocrinology, Diabetes and Metabolism, University of Arizona College of Medicine - Phoenix, Phoenix, Arizona, USA, and ${ }^{4}$ Carl T Hayden Phoenix VA Medical Center, Phoenix, Arizona, USA

\author{
Correspondence \\ should be addressed \\ to A Gandhi \\ Email \\ gandhiad4@gmail.com
}

\section{Summary}

Immobilization-induced hypercalcemia is an uncommon cause of elevated calcium which is usually diagnosed following extensive systemic workup and exclusion of more common etiologies. Previously reported cases have largely described this phenomenon in adolescents and young adults a few weeks to months after the initial onset of immobilization. Metabolic workup tends to demonstrate hypercalcemia, hypercalciuria, and eventual osteoporosis. While the exact mechanism remains largely unclear, a dysregulation between bone resorption and formation is central to the pathogenesis of this disease. Decreased mechanical loading from prolonged bedrest tends to increase osteoclast induced bone resorption while promoting osteocytes to secrete proteins such as sclerostin to reduce osteoblast mediated bone formation. We describe the case of an 18-year-old male who was admitted following intraabdominal trauma. He underwent extensive abdominal surgery including nephrectomy resulting in initiation of dialysis. After 6 months of hospitalization, the patient gradually began developing uptrending calcium levels. Imaging and laboratory workup were unremarkable for any PTH-mediated process, malignancy, thyroid disorder, adrenal disorder, or infection. Workup did reveal significant elevated bone turnover markers which in combination with the clinical history led the physicians to arrive at the diagnosis of immobilization induced hypercalcemia. In order to prevent decreased rates of bone loss, the patient was administered denosumab for treatment. Hypocalcemia followed treatment expectedly and was repleted with supplementation via the patient's total parenteral nutrition.

\section{Learning points:}

- Immobilization-induced hypercalcemia should remain as a differential diagnosis of patients with prolonged hospitalizations with hypercalcemia.

- Extensive workup of common etiologies of hypercalcemia should be considered prior to arriving at this diagnosis.

- Denosumab, while off-label for this usage, offers an effective treatment option for immobilization-induced hypercalcemia though it carries a risk of hypocalcemia especially among patients with renal disease.

\section{Background}

This case highlights the diagnosis and management of an uncommon cause of hypercalcemia that may often be overlooked by other more common etiologies. It may best be considered after more prevalent conditions that induce hypercalcemia have been ruled out such as primary hyperparathyroidism and malignancy. Treatment tends to be variable and unique to each patient's presentation though it typically revolves around i.v. hydration, diuresis, 
glucocorticoids, and denosumab therapy. In our patient, denosumab was chosen to reduce the rate of bone turnover due to this disorder, as the patient was likely going to be persistent with the functional limitations. An expected but severe side effect of hypocalcemia resulted after treatment which was corrected with supplementation.

\section{Case presentation}

The patient is an 18-year-old male with no prior medical history was admitted after suffering a gunshot wound in the abdomen. He underwent extensive intraabdominal surgery with bilateral nephrectomy, bowel resection, and splenectomy. The patient required initiation of dialysis following nephrectomy. His hospital course was complicated by subsequent gastrointestinal bleeding, respiratory failure, retroperitoneal abscess, acalculous cholecystitis, and pericardial effusion requiring window procedure. The patient was normocalcemic on admission but gradually displayed uptrending calcium levels starting approximately 2 months into admission to reach a peak of $16.3 \mathrm{mg} / \mathrm{dL}(\mathrm{nl}: 8.8-10.4 \mathrm{mg} / \mathrm{dL})$ at 4 months. Over the following 2 months the patient's calcium level normalized without intervention but he started to become hypercalcemic once again prompting endocrinology consultation. At this time point, 6 months into admission, a hypercalcemia workup was initiated.

\section{Investigation}

The patient's labs (Table 1) were notable for parathyroid hormone (PTH) $11 \mathrm{pg} / \mathrm{mL}$ (nl: 15-65 pg/mL), parathyroid hormone related peptide (PTHrP) $44 \mathrm{pg} / \mathrm{dL}$ (nl:14-27 $\mathrm{pg} / \mathrm{dL}), 25 \mathrm{OH}$ vitamin D $19 \mathrm{ng} / \mathrm{mL}(\mathrm{nl}:>20 \mathrm{ng} / \mathrm{mL}$ ), $1,25 \mathrm{OH}$ vitamin $\mathrm{D}<8 \mathrm{pg} / \mathrm{mL}$ (nl: $18-72 \mathrm{pg} / \mathrm{mL}$ ), and collagen type I C-telopeptide (CTx) $>6000 \mathrm{pg} / \mathrm{mL}$ (nl: 435-2924 pg/mL). Laboratory evaluation for human immunodeficiency virus (HIV), hyperthyroidism, multiple myeloma, and adrenal insufficiency was all negative. No calcium was being administered through the patient's total parenteral nutrition (TPN). A low calcium bath was being used for dialysis. The patient endorsed a diet with minimal consumption of dairy and other calcium rich products. The patient did not have any prior history of malignancy and extensive previous imaging of the patient's chest, abdomen, pelvis, brain, and spine did not reveal any indication of such. Bone mineral density testing was not performed during the admission. Based on the comprehensive workup, it was determined that the patient's hypercalcemia was most likely secondary to his prolonged immobilization.

\section{Treatment}

Given the diagnosis of immobilization-induced hypercalcemia, a therapeutic intervention of denosumab was selected in order to reduce the rate of bone turnover. The patient was given a dose of denosumab $60 \mathrm{mg}$ s.c. once for treatment. Expectedly, following treatment, the patient experienced significant decline in calcium and phosphorus levels to nadir $7.3 \mathrm{mg} / \mathrm{dL}(\mathrm{nl}: 8.8-10.4 \mathrm{mg} / \mathrm{dL}$ ) and $0.9 \mathrm{mg} /$ dL (nl: $2.7-5.9 \mathrm{mg} / \mathrm{dL}$ ), respectively. Supplementation was initiated with cholecalciferol $0.25 \mu \mathrm{g}$ BID, calcium

Table 1 Laboratory workup.

\begin{tabular}{l} 
Laboratory blood tests \\
\hline Collagen type I C-telopeptide $(\mathrm{pg} / \mathrm{mL})$ \\
HIV $1 / 2$ Ag/Ab screen (4th Gen) \\
Alkaline phosphatase, bone-specific $(\mu \mathrm{g} / \mathrm{L})$ \\
Cortisol level ( $\mu \mathrm{g} / \mathrm{dL})$ \\
AM \\
Random (baseline) \\
30 min post stimulation \\
60 min post stimulation \\
90 min post stimulation \\
Thyroid stimulating hormone $(\mu \mathrm{IU} / \mathrm{mL})$ \\
PTH, intact (pg/mL) \\
PTH, related protein (pg/mL) \\
Random \\
Pre-dialysis \\
Post-dialysis \\
Vitamin D 25, OH (ng/mL) \\
Vitamin D 1,25 OH total $(\mathrm{pg} / \mathrm{mL})$
\end{tabular}

\begin{tabular}{c}
\hline Result \\
\hline$>6000$ \\
Non-reactive \\
23.1 \\
16.4 \\
5.7 \\
13.6 \\
18.1 \\
19.6 \\
1.64 \\
11 \\
44 \\
80 \\
73 \\
15.7 \\
$<8$ \\
\hline
\end{tabular}

\begin{tabular}{c}
\hline Reference range \\
\hline $435-2924$ \\
- \\
$13.7-128.0$ \\
$4.8-19.5$ \\
$2.5-19.5$ \\
- \\
- \\
- \\
$0.30-4.00$ \\
$15-65$ \\
$14-27$ \\
$14-27$ \\
$14-27$ \\
$>20$ \\
$19-83$ \\
\hline
\end{tabular}

HIV, human immunodeficiency virus; PTH, parathyroid hormone. 
carbonate $1000 \mathrm{mg}$ QID, and calcium gluconate $2 \mathrm{mEq}$ via the patient's TPN. The patient's hemodialysis bath was adjusted for this fluctuation in calcium level as well.

\section{Outcome and follow-up}

The patient's calcium and phosphorus level slowly normalized over the following days and the patient was eventually discharged to a skilled nursing facility with continued electrolyte monitoring and nutrient supplementation.

\section{Discussion}

Hypercalcemia carries a broad differential diagnosis that accompanies both parathyroid-mediated and nonparathyroid-mediated forms (1). Hypercalcemia, as a whole, can be seen in rates of over $4 \%$ of hospitalized patients (2). Immobilization tends to be a less common etiology of hypercalcemia though diagnosis remains important for appropriate management of this disease. Elevated calcium levels have widespread clinical implications including decreased QT-interval, bradycardia, neuropsychiatric disturbances, such as depression and cognitive dysfunction, as well as both acute and chronic renal insufficiency (3). Previous literature has demonstrated cases of immobilization induced hypercalcemia among patients with spinal cord injury $(4,5,6)$, traumatic fractures $(7,8)$, and Guillan Barre syndrome (9). The development of this disease tends to occur on average after a few months (10) after the onset of immobilization though in some cases it can develop sooner (8). An exhaustive exploration of etiologies is important to rule out before reaching this diagnosis including adrenal insufficiency, thyroid disorders, malignancy, hyperparathyroidism, and nutrition-induced. In our patient, after an extensive exploration of other etiologies, the diagnosis of immobilization induced hypercalcemia was arrived upon. The presence of bone-specific biomarkers such as CTx supported our diagnosis. The patient's elevated PTHrP was thought to be secondary to his renal insufficiency. A variety of treatment modalities have been used in previous cases for immobilization induced hypercalcemia. Due to the patient's renal insufficiency in the setting of bilateral nephrectomy, denosumab was selected as the primary treatment modality. Denosumab has been well described in literature as a potential treatment option in similar patients with immobilization hypercalcemia and renal insufficiency (11). It should be noted that though despite its efficacy in immobilization-induced hypercalcemia it is currently considered an off-label usage and not a primary indication approved by the FDA. An expected hypocalcemic state was induced following treatment. This is a known side effect of denosumab especially in those with renal insufficiency due to maintenance of serum calcium levels being mediated by bone resorption (12). The patient's hypocalcemia was able to be quickly corrected using calcium and vitamin D supplementation and remained persistently normal throughout the remainder of his hospital course.

Declaration of interest

The authors declare that there is no conflict of interest that could be perceived as prejudicing the impartiality of the research reported.

\section{Funding}

This study did not receive any specific grant from any funding agency in the public, commercial or not-for-profit sector.

\section{Patient consent}

Written informed consent for publication of their clinical details was obtained from the patient.

\section{Author contribution statement}

Anand Gandhi was the primary author of the manuscript involved with review of patient information, composition of the manuscript, evaluation of literature. Michael Mortensen, Sonie Sunny, and Pawarid Techathaveewat were responsible for the acute care and management of the patient and contributed significant revisions to the manuscript. Jerome Targovnik contributed significant revisions to the manuscript. Mahmoud Alsayed was the primary attending physician responsible for the care of the patient who contributed significantly to manuscript revision and generation.

\section{References}

1 Carroll MF \& Schade DS. A practical approach to hypercalcemia. American Family Physician 200367 1959-1966.

2 Catalano A, Chila D, Bellone F, Nicocia G, Martino G, Loddo I, Morabito N, Benvenga S \& Loddo S. Incidence of hypocalcemia and hypercalcemia in hospitalized patients: is it changing? Journal of Clinical and Translational Endocrinology 201813 9-13. (https://doi. org/10.1016/j.jcte.2018.05.004)

3 Shane E \& Irani D. Hypercalcemia: pathogenesis, clinical manifestations, differential diagnosis, and management. In Primer on the Metabolic Bone Diseases and Disorders of Mineral Metabolism, 6th ed., pp. 176-180. Ed MJ Favus. Washington, DC: American Society for Bone and Mineral Research, 2006.

4 Massagli TL \& Cardenas DD. Immobilization hypercalcemia treatment with pamidronate disodium after spinal cord injury. Archives of Physical Medicine and Rehabilitation 199980 998-1000. (https://doi. org/10.1016/s0003-9993(99)90050-3)

5 Maynard FM \& Imai K. Immobilization hypercalcemia in spinal cord injury. Archives of Physical Medicine and Rehabilitation 197758 16-24. 
6 Carey DE \& Raisz LG. Calcitonin therapy in prolonged immobilization hypercalcemia. Archives of Physical Medicine and Rehabilitation 198566 640-644.

7 Hyman LR, Boner G, Thomas JC \& Segar WE. Immobilization hypercalcemia. American Journal of Diseases of Children 1972124 723-727. (https://doi.org/10.1001/archpedi.1972.02110170101017)

8 Wolf AW, Cuinard RG, Riggins RS, Walter RM \& Depner TH. Immobilization hypercalcemia: a case report and review of the literature. Clinical Orthopaedics and Related Research 1976118 124-129. (https://doi.org/10.1097/00003086-19760700000021)

9 Clouston WM \& Lloyd HM. Immobilization-induced hypercalcemia and regional osteoporosis. Clinical Orthopaedics and Related Research
1987216 247-252. (https://doi.org/10.1097/00003086-19870300000038)

10 Meythaler JM, Tuel SM \& Cross LL. Successful treatment of immobilization hypercalcemia using calcitonin and etidronate. Archives of Physical Medicine and Rehabilitation 199374 316-319.

11 Booth KA \& Hays CI. Using denosumab to treat immobilization hypercalcemia in a post-acute care patient. Journal of Clinical Endocrinology and Metabolism 201499 3531-3535. (https://doi. org/10.1210/jc.2013-4205)

12 Laskowski LK, Goldfarb DS, Howland MA, Kavcsak K, Lugassy DM \& Smith SW. A RANKL wrinkle: denosumab-induced hypocalcemia. Journal of Medical Toxicology 201612 305-308. (https://doi.org/10.1007/ s13181-016-0543-y)

Received in final form 29 August 2021

Accepted 1 September 2021 